\title{
Progress in the Search for Dark Matter Using Upward-going Muons in NOvA
}

\author{
A. Tsaris*, L. Aliaga, P. Ding, A. Norman \\ Fermi National Accelerator Laboratory \\ E-mail: atsarisefnal.gov \\ C. Group, S. Kurbanov, R. Mina, Y. Oksuzian, C. Principato \\ University of Virginia
}

\begin{abstract}
The NOvA collaboration has constructed a 14,000 ton, fine-grained, low-Z, total absorption tracking calorimeter at an off-axis angle to an upgraded NuMI neutrino beam. This detector, with its excellent granularity, energy resolution and relatively low-energy neutrino thresholds, was designed to measure $v_{\mu} \rightarrow v_{e}$ neutrino appearance. These characteristics combined with the detector's timing resolution, continuous readout structure, and placement on the surface of the Earth make it ideal for use as a neutrino telescope. In order to operate in this telescope mode, NOvA experiment has developed a highly efficient upward-going muon trigger which is able to achieve a background suppression rate of $10^{4}$ within the experiment's DAQ systems which is then increased by an additional factor of $10^{6}$ in the offline to fully suppress the flux of downward going cosmics rays. The trigger efficiencies and rejection capabilities allow NOvA to be competitive in indirect dark matter searches for low-mass WIMPs. We present initial progress and results for an upward going muon analysis using data-driven background estimations that include signal and sideband region validation, as well as timing based directional track reconstruction. In addition we present results from the analysis of muon depletion corresponding to the celestial position of the moon, as a technique to determine an upper limits on the pointing resolution of the NOvA detector.
\end{abstract}

38th International Conference on High Energy Physics

3-10 August 2016

Chicago, USA

* Speaker. 


\section{Introduction}

Weakly interactive massive particles (WIMPs) captured by the gravitational field of the Sun that are slowed through collisions with solar matter can accumulate in the solar core. WIMP annihilation in the solar core can then produce final state neutrinos with energies much larger than the normal solar neutrino spectrum. The signal from these types of annihilation processes would be characterized by an excess of high-energy $(>0.5 \mathrm{GeV})$ neutrino events pointing back to the Sun $[1,2]$. In the NOvA detector, these types of events could be isolated and detected through measurement of the upward going muon flux from charged current $v_{\mu}$ interactions occurring either within the NOvA detector or in the rock below the detector. The large and massive NOvA far detector is an ideal tool for searching for these types of dark matter signatures due to its fine granularity, precise timing information, energy resolution and low single channel detection threshold. These features combine to provide both high efficiency for the detection of muon tracks and muon neutrino interactions and excellent point resolution and directionality determination for the tracks.

The NOvA detector is located on the surface with only a $3 \mathrm{~m}$ earth equivalent overburden. As a result, the upward going muon flux needs to be identified and selected at the trigger level in the experiment to separate it from the downward going cosmic ray muon flux. Due to the minimal overburden of the NOvA detector hall, the downward-going muon rate in the NOvA far detector is approximately $150,000 \mathrm{~Hz}$ over the surface of the detector. This background flux is suppressed by more than a factor of $10^{4}$ at trigger level to an overall rate of less than $10 \mathrm{~Hz}$. The selections applied at trigger level are minimally biasing to the data and result in an initial selection efficiency of better than $85 \%$ in the range of muon momenta from $0.5-5 \mathrm{GeV} / \mathrm{c}$.

The neutrino flux from dark matter annihilation is highly model dependent and can produce neutrinos with energies ranging from $\sim 0.5 \mathrm{GeV}$ to many $\mathrm{TeV}$ based on the masses of the WIMPs. In all of these cases the NOvA detector is sensitive to the resulting interactions and can perform the reconstruction of the events. In the high-mass portion of the model space, NOvA is limited by its size to the flux and is not as sensitive as larger experiments like the IceCube detector [3] to the ultra high energy signatures. For lower WIMP mass scenarios $\left(M_{\text {Wimp }}<\sim 20 \mathrm{GeV}\right)$, the SuperKamiokande experiment currently has the best sensitivity [4, 5] for indirect dark matter detection. The efficient of NOvA's upward-going muon trigger and cosmic ray background rejection allow NOvA to be competitive with Super-Kamiokande in this low mass regime, and to be particular sensitive to fluxes with final state signatures below $1 \mathrm{GeV}$.

A particular advantage that NOvA has, compared to past experiments that have performed similar dark matter annihilation searches, is the experiment's low energy threshold for muon reconstruction. A $1 \mathrm{GeV}$ muon travels approximately 5 meters in the NOvA detector and can leave visible energy in excess of 120 detection cells. The low-Z nature of the detector combined with the fine grained, high sampling multiplicities associated with single particles result the ability to detect and reconstruct neutrino interactions at thresholds well below $1 \mathrm{GeV}$.

The challenge for the upward going muon dark matter search is combining the trigger efficiency for these low-energy interactions with the timing information for each of the hits in the interaction. In the case of long tracks, the timing information is aggregated across all the hits in the track and the physical size length of the track provides a long lever arm for determination of the track's directionality. For shorter tracks, the timing information will not be as powerful for 
rejecting downward-going backgrounds. Using stopping or fully-contained events and using the top and sides of the detector to veto downward-going events can provide an additional two orders of magnitude rejection.

This analysis uses a powerful data-driven control region technique that is used for estimating the backgrounds to the dark matter search. In this method a measurement is made of the upwardgoing muon flux during the day when the Sun is above the horizon and can not possibly be the source for the events. This "day" sample is then used to fully describe the expected background and scaled by the exposure to determine the proper re-weighting and background contamination that is expected in the corresponding "night" sample. The technique then performs the actual signal search during the "night" when the Sun is below the horizon and looks for an excess in weighted distribution. Using this method, a decomposition of the background need not be performed. Decomposing the background requires a full understanding of the atmospheric neutrino sample which will take longer for us to complete, but will open the door to atmospheric neutrino physics and to further optimization of this search.

\section{Pointing Resolution}

The upward going muon analysis relies on correlating the direction of neutrino interactions with the position of a celestial object. This correlation is limited by the pointing resolution of the detector and its reconstruction algorithms. To determine an upper limit on the pointing resolution of the NOvA detector, the flux of downward going muons was correlated with the position of the moon. The resulting deficit in the spectrum was then unfolded to extract an effective resolution function for the NOvA detector that represents the smearing of track directions due to the detector's geometry, reconstruction algorithms and deflection of tracks by the magnetic field of the earth at the latitude of the detector. This technique has been used by other experiments including MINOS [6], IceCube [7] and MACRO [8].

For this analysis we require muon tracks at least $15 \mathrm{~m}$ long and within $5^{\circ}$ of the position of the Moon. Figure 1 shows the differential density of the cosmic rays seen by the NOvA Far Detector as a function of the angular distance between the track direction and the position of the Moon $(\Delta \theta)$. A deficit is observed in the region corresponding to the angular size of the Moon, $\Delta \theta<1.5^{\circ}$ and a flat distribution for $\Delta \theta>1.5^{\circ}$. Eq. 2.1 models the blocking function for the flux distribution with a two dimensional Gaussian smearing [6], where $\lambda$ is the the average differential muon flux and $R_{m}=0.26^{\circ}$ is the angular radius of the Moon. The parameter $\sigma$ represents the smearing and is estimated by fitting the data to Eq. 2.1. For the NOvA detector we determined $\sigma=1.23^{\circ}$.

$$
\frac{\Delta N}{\Delta \Omega}=\lambda\left[1-\frac{R_{m}^{2}}{2 \sigma^{2}} e^{-\Delta^{2} \theta / 2 \sigma^{2}}\left(1+\frac{\left(\Delta^{2} \theta-2 \sigma^{2}\right) R_{m}^{2}}{8 \sigma^{4}}+\frac{\left(\Delta^{4} \theta-8 \Delta^{2} \theta \sigma^{2}+8 \sigma^{4}\right) R_{m}^{4}}{192 \sigma^{8}}\right)\right]
$$

\section{Upward-going Muon Analysis}

The data used in the upward going muon analysis was acquired using data driven software trigger that examined in real-time the NOvA readout stream[9] for upward going muon topologies. 


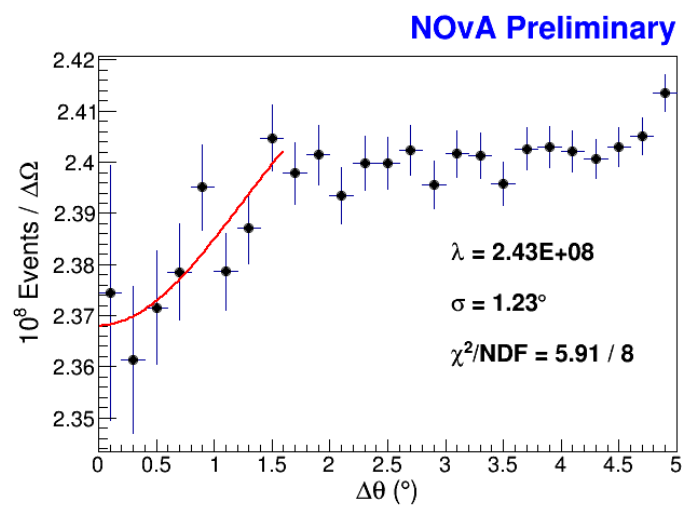

Figure 1: Cosmic rays flux at the NOvA Far Detector as a function of the angle to the Moon position. The red curve shows the result of fitting the distribution.

The upward-going muon trigger started taking data on Dec 12th, 2014. The trigger is based on a fast track reconstruction and a log-likelihood discriminator (LLR) which uses the timing information of the hits to separate tracks into two populations based on an upward or downward going hypothesis [10].

The WIMP search uses a blind analysis scheme to prevent bias. This is implemented through a multi-level blinding, where the signal box is defined both in the angular position of the Sun with respect to the horizon, the angular deviation of the particle tracks in relation to the Sun's altitude and azimuth and the log-likelihood discriminator of the tracks. The primary blind is placed on all data with the Sun below $10^{\circ}$ of the local horizon (denoted "night") and the secondary blind as the data in this region where the tracks to $\operatorname{Sun} \Delta \theta$ is less than $10^{\circ}$. A near side band ("twilight") region to the primary blind is placed between $\pm 10^{\circ}$ to the local horizon.

Stability of the detector is established and checked using zero bias, downward going, cosmic ray data. Checks of relevant reconstruction and kinematics observables agree in their rates and shapes agree to $1 \%$ or better when comparing day and night samples. Figure 2 shows the agreement between the track associated hit multiplicities and direction cosine $(\mathrm{Z})$ distributions. Similarly event selection efficiencies across all selection variables were checked to agree at a level of better than $1 \%$.

The high latitude of the NOvA detector results in a highly asymmetric (in altitude) path for the Sun across the detector's local sky. As a result, the verification of no day/night bias in the angular deviation variable $(\Delta \theta)$ between track and Sun was performed using a randomized re-weighting procedure. In this technique the data was split into day/night samples. Then each cosmic ray in the night sample was assigned the true position of the Sun, while each cosmic ray in the day sample assigned a random position for the Sun that corresponded to a point along the Sun's trajectory during the corresponding night period of the same day. The $\Delta \theta$ was then calculated either between the track and the true position of the Sun or the "phantom" position. In this manner the ensemble distributions, which should show no correlation between a downward going cosmic ray flux and a Sun that is shielded by the Earth, are correctly re-weighted in their shape and can be compared.

Using the 'phantom' position of the Sun in the day control region we compare the $\Delta \theta$ distribution for the day and night samples of the cosmic rays. This is shown in Figure 3. We observe a 

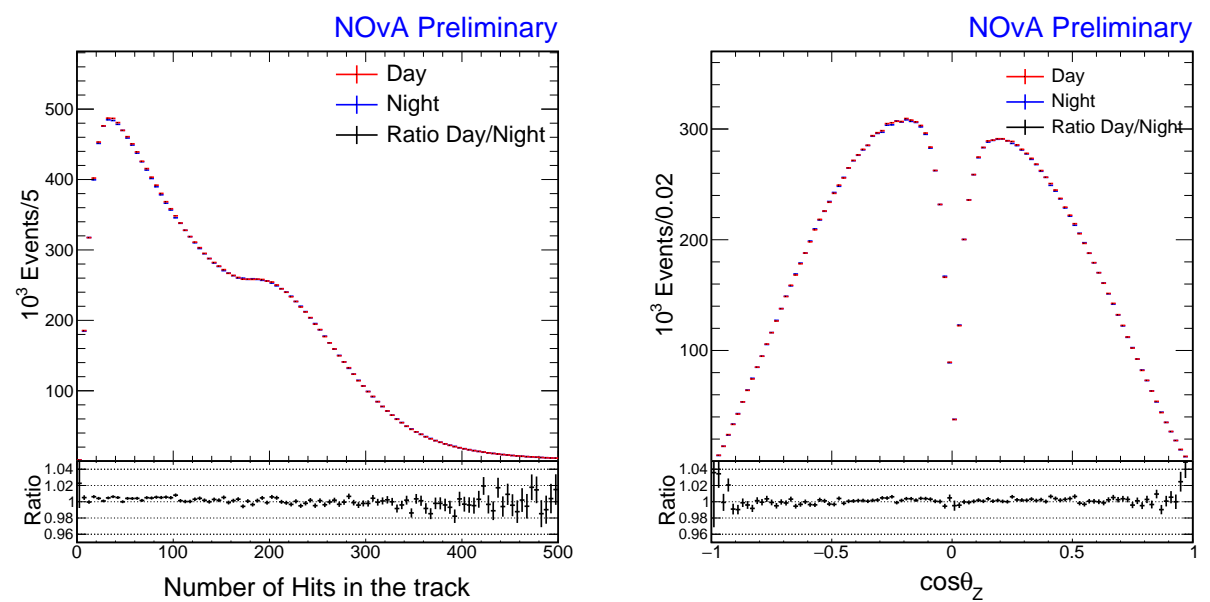

Figure 2: Track associated hit multiplicity and direction cosine $(Z)$ distributions for day/night cosmic ray fluxes. The distributions for day and night agree to better than $1 \%$.

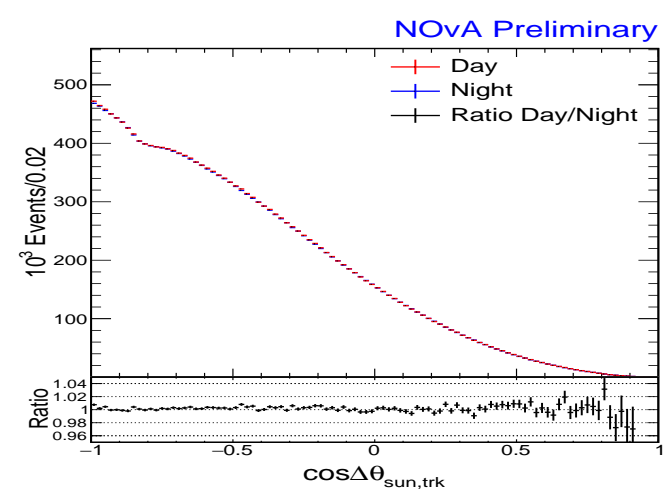

Figure 3: Distribution of $\cos (\Delta \theta)$, where $\theta$ is the angular separation between track and Sun. Day sample uses a randomized reweighting technique for the solar position.

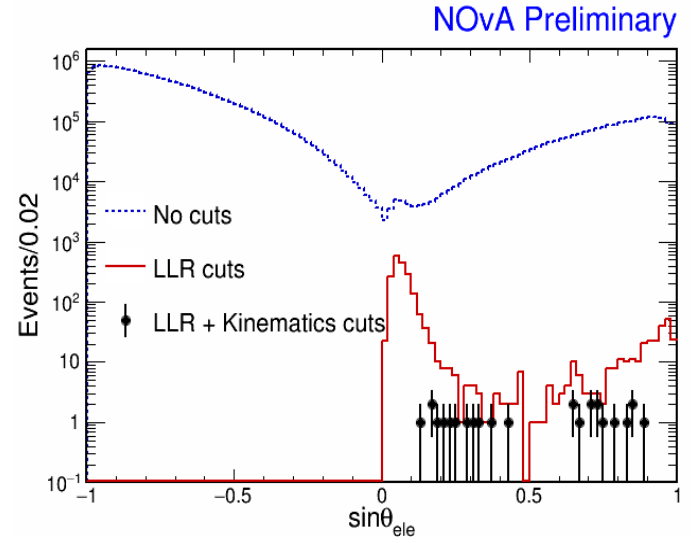

Figure 4: The distribution of sine of the elevation angle for each track (blue), after applying the timing cuts (red) and after applying the full set of cuts for this analysis (black). Negative elevation angles indicates a downward-going track. The current distribution represents 77 days of live time.

weak systematic trend that changes the ratio by about $2 \%$ over the range of the distribution. This asymmetry thought to be due to the differing attenuation of cosmic rays in the atmosphere with respect to day/night temperature changes. However no attempt is made to correct for it, and the effect is taken as a systematic uncertainty on the analysis.

For the upward-going track sample, a signal free control region (day), is used to evaluate the impact of the selection criteria and verify the event reconstruction. In particular the near sideband ("twilight" region) is used to evaluate the systematic related to the determination of track direction 
for near horizontal muons and log-likelihood uncertainties dependence on track elevation.

\section{Data Driven Background Estimates}

Background estimation for the through going muon analysis was performed by applying all selections cuts to the cosmic ray sample. Then the log-likelihood ratio for each track was computed. The efficiency for the cuts was determined from the minimum bias cosmic ray sample. In this process all of the muons from the minimum bias sample are considered to be "signal" like in their track characteristics so that these efficiencies apply also to the upward going tracks (i.e. the difference between upward and downward going tracks is their timing distributions and $\mathrm{dE} / \mathrm{dx}$ profiles, but not their physical extent or $\chi^{2}$ distributions on the geometric fits). These studies measured a signal efficiency of $30 \%$ for "golden" tracks. Optimization was not performed in the initial analysis to relaxation the minimum track length selection cut. Relaxing this cut significantly increase the signal efficiency and is planned for future analysis. The efficiencies related to the cuts that select the upward going track direction were estimated by inverting the LLR discriminator when applied to the downward-going cosmic-ray muons. This was done by first applying baseline clean up cuts to the sample and then we applying the timing cuts separately.

Figure 4 shows the elevation angle distribution for upward-going triggered events in the daytime control sample. Applying the timing selections results in rejecting the majority of the downwardgoing muons. Tracks passing those timing cuts are predominantly horizontal or slightly upwardgoing (red curve). The abundance of mostly-horizontal tracks in this subsample is explained by the position of the far detector on the surface. Slightly upward-going cosmic ray-induced muons arise due to scattering in the detector or the material surrounding the detector hall. At steeper track angles the detector shielding is sufficient to attenuate this effect and explains the fall-off in the elevation angle distribution.

After applying all the selection criteria 33 tracks remained as upward-going muon candidates, correspond to 77 days of live time. Normalizing for the day/night exposure this is extrapolated to the prediction for the unblinded signal region. This was checked for the near sidebands. A prediction for the twilight region of $23 \pm 5$ background was made. A total of 24 events was observed in the region.

\section{Summary}

This work demonstrates that NOvA is capable of isolating a sample that is likely rich in neutrino-induced upward-going muons and opens the door to a competitive dark matter search. Furthermore, we have demonstrated NOvA's ability to point to celestial objects. Finally, we have used a control sample of cosmic-ray muons to demonstrate that the NOvA detector performance is stable comparing day versus night operations.

\section{References}

[1] J. S. Hagelin, K. W. Ng and K. A. Olive, Phys. Lett. B 180, 375 (1986).

[2] J. Buckley, D. F. Cowen, S. Profumo, A. Archer, M. Cahill-Rowley, R. Cotta, S. Digel and A. Drlica-Wagner et al., arXiv:1310.7040. 
[3] M. G. Aartsen et al. [IceCube Collaboration], Phys. Rev. Lett. 110, no. 13, 131302 (2013).

[4] T. Tanaka et al. [Super-Kamiokande Collaboration], Astrophys. J. 742, 78 (2011).

[5] K. Choi et al. [Super-Kamiokande Collaboration], Phys. Rev. Lett. 114, no. 14, 141301 (2015).

[6] P. Adamson et al. [MINOS Collaboration], Astropart.Phys.34:457-466, 2011.

[7] M.âĂL'G. Aartsen et al. [IceCube Collaboration], Phys. Rev. D 89, 102004.

[8] M. Ambrosio et al. [MACRO Collaboration], Phys. Rev. D 59, 012003.

[9] M. Fischler, C. Green, J. Kowalkowski, A. Norman, M. Paterno and R. Rechenmacher, J. Phys. Conf. Ser. 396, 012020 (2012).

[10] R. Mina et al. [NOvA Collaboration], J. Phys. Conf. Ser. 664, 082034 (2015), arXiv:1510.07571. 